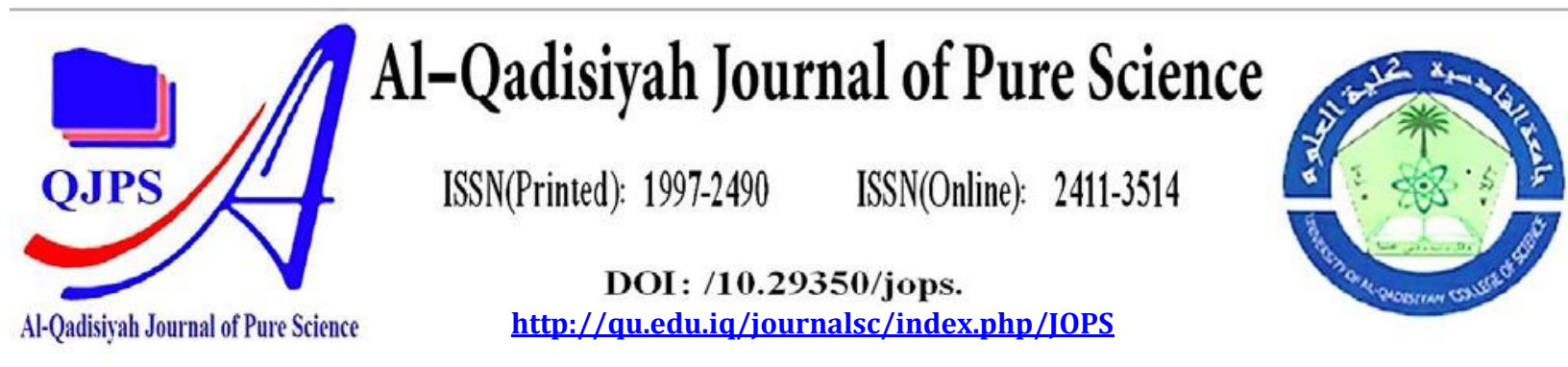

\title{
Solving Riccati type $q q$-Difference Equations via Difference Transform Method
}

\section{Authors Names \\ a: Ahmed Y. Abdulmajeed \\ b: Ayad R. Khudair \\ Article History \\ Received on: 2/6/2021 \\ Revised on: 15/7/2021 \\ Accepted on:25/7/2021 \\ Keywords: \\ Time scales calculus; Differential transform method; Riccati type $q$-difference equations \\ DOI:https://doi.org/10.293 \\ 50/jops. 2021.26. 4.1318}

\section{ABSTRACT}

In this paper, we deal with a time scale that its delta derivative of graininess function is a nonzero positive constant. Based on the Taylor formula for this time scale, we investigate the difference transform method (DTM). This method has been applied successfully to solve Riccati type $q$-difference equations in quantum calculus. To demonstrate the ability and efficacy of this method, some examples have been provided.

\section{Introduction}

One of the simplest and more important type of nonlinear differential equations are Riccati differential equations [32]. Due to their close connection to the Bessel function, these equations have often appeared in many physical problems, likes; static Schrödinger equation [12], Newton's laws of motion [29], 3DGross-Pitaevskii equation [26], cosmology problem [33]. Also, it relates to many mathematical subjects, including; projective differential geometry [2], calculus of variations [41], optimal control [30], and dynamic programming [6]. Several techniques have been used to solve constant coefficients Riccati differential equations, such as; operation matrix method [31], variational iteration method [17], polynomial least squares method [9], homotopy perturbation method [1], Legendre wavelet method [3], and Adomian's decomposition method[15].

Riccati difference equations are not different from Riccati differential equations, as they have many applications in various fields. Where it arises in the filtering problem [27], the optimal control problem [5] and it has been studied by numerous scholars [4, 42, 38, 28]. In fact, the first study appeared on the difference Riccati equations was in 1905 by H. Tietze [39]. 
The q-difference equation (q-DEs)is a type of difference equation that is based on q-calculus. Indeed, the old references refer to the beginning of q-calculus was in the late 20th century to make links between mathematics and physics [16]. It has a variety of uses in mathematics, engineering and science, including basic hypergeometric functions [10,11,36, 35, 34], orthogonal polynomials [25], combinatorics [19], and quantum theory [21]. In recent years, several scholars have attempted to solve many types of q-DEs by using semi-analytic methods, including; the q-differential transformation method (q-DTM)[13, 14], variational iteration method [40], succes- sive approximation method, and homotopy analysis method [37].

In this paper, we deal on time scale $\mathbb{T T}$ that its delta derivative of graininess function is a nonzero positive constant, that is $\mu^{\Delta}=\zeta \zeta>0$. Based on Taylor formula for this time scale, we introduce some fundamental theorems related to DTM in order to solve the following Riccati type $q$-difference equations on the time scale $\mathbb{T} \mathbb{T}=\overline{q^{\mathbb{N}}}=\{0\} \bigcup \quad\left\{q^{t} \mid t \in \mathbb{N}, 0<q<1\right\}$ :

$$
\Psi \Delta(t)=g_{1}(t) \Psi(t)+g_{2}(t) \Psi(t)^{2}+g_{3}(t), \quad \Psi(t)=A .
$$

where $g_{c}(t), r=1,2,3$ are analytic function on $\mathbb{T T}$.

\section{Preliminaries}

This section has provided a brief overview of time scale preliminary information and their relationship to q-calculus

Definition 2.1 [18] A time scale is a non-empty arbitrary closed subset of real numbers denoted by TT. Time scale examples, $[0,1]$, the natural numbers set $\mathbb{N}$, the real numbers set $\mathbb{R},[0,1] \cup \quad[2,3]$ and the cantor set whereas the set of rational numbers $\mathbb{Q}$ complex numbers $\mathbb{C}$, and $[0,1),(0,1],(0,1),(0,1] \cup\{2 b-a\}$ are not time scales.

Definition 2.2 [8] Let TT be any time scale and $r \in \mathbb{T T}$. Operator of a forward jump $\sigma: \mathbb{T T} \rightarrow \mathbb{T T}$ is given as:

$$
\sigma(r)=\inf \{s \in \mathbb{T} \mathbb{T}: s>r\}
$$

while, the Operator of a backward jump $\rho: \mathbb{T T} \rightarrow \mathbb{T T}$ at all $r \in \mathbb{T T}$ is given as:

$$
\rho(r)=\sup \{s \in \mathbb{T} \mathbb{T}: s<r\}
$$

Definition 2.3 [7]

For $s \in \mathbb{T T}$, the function $\mu$ : $\mathbb{T} \rightarrow[0, \infty)$ defined by

$$
\mu(s)=\sigma(s)-s
$$

is called graininess function.

assume that

$$
\mathbb{T T}=\bar{q}^{\mathbb{N}}=\left\{q^{t} \mid t \in \mathbb{N}, 0<q<1\right\} \cup\{0\}
$$

Let the $q$-shift factorial is given by

$$
(t ; q)_{0}=1 \quad \text { and } \quad(t ; q)_{m}=\prod_{j j=0}^{m-1}\left(1-a q^{j j}\right), \quad m=1, \ldots, m
$$

such that $t$ is real number. 
Definition 2.4 [7] For $t \in \overline{q^{\mathbb{N}}}$, the delta $q$-derivative of a function $g(t)$ on $\mathbb{T} \mathbb{T}=\overline{q^{\mathbb{N}}}$ is given by

$$
g^{\Delta}(t)= \begin{cases}\frac{g g(q t)-g g(t)}{(q-1) t}, & \text { iiff } t \in \overline{q^{\mathbb{N}}} \\ \lim _{n \rightarrow \infty} \frac{g g\left(q^{n}\right)-g g(0)}{q^{n}}, & \text { iiff } t=0\end{cases}
$$

Definition 2.5 [22] Let $G: \overline{q^{\mathbb{N}}} \rightarrow \mathbb{R}$ a pre-antiderivative of the function $g: \overline{q^{\mathbb{N}}} \rightarrow \mathbb{R}$.such that $G^{\Delta \Delta}(t)=g(t)$. The indefinite integral of the function $g$ is define by

$$
\int g(t) \Delta t=G(t)+c
$$

where $c$ is a constant. Moreover, the definite integral is defined by

$$
\int_{b}^{d d} g(t) \Delta t=G(d)-g(b), \forall b, d \in \overline{q^{\mathbb{N}}}
$$

Definition 2.6 [8] The monomials $h_{n}: \mathbb{T T} \times \mathbb{T T} \rightarrow \mathbb{R}, \quad n \in \mathbb{N}_{0}$ on a time scale $\mathbb{T} \mathbb{T}$ are defined by

$$
\begin{gathered}
h_{0}(r, s)=1 \\
h_{n+1}(r, s)=\int_{s}^{r} h_{n}(r, s) d r, \quad n \in \mathbb{N}, r, s \in \mathbb{T} \mathbb{T} .
\end{gathered}
$$

Hence, the $\Delta$-derivative of $h_{n}(r, s)$ with respect to $r$ given by

$$
h_{n}^{\Delta}(r, s)=h_{n-1}(r, s), n \geq 1
$$

Example 2.1 [8]

1. When $\mathbb{T} \mathbb{T}=\overline{q^{\mathbb{N}}}$, we get

$$
h_{n}(t, s)=\prod_{\omega=0}^{n-1} \frac{t-s q^{\omega}}{\sum_{n=0}^{\omega} q^{n}}, \forall n \in \mathbb{N}
$$

2. When $\mathbb{T} T=\mathbb{R}$, we get

$$
h_{n}(t, s)=\frac{(t-s)^{n}}{n !}, \quad \forall n \in \mathbb{N}
$$

3. When $\mathbb{T T}=\mathbb{Z}$, we get

$$
h_{n}(t, s)=2^{t-s} \gg \forall n \in \mathbb{N}
$$

Theorem 2.1 [8] For all $t, s \in \mathbb{T T}$ and $j j \in \mathbb{N}_{0}$, we have

$$
0 \leq h(t, s) \leq \frac{(t-s)^{j j}}{j j !}, \quad \forall r \geq s
$$

Let $m \in \mathbb{N}$ and $g: \mathbb{T} \rightarrow \mathbb{R}$ is $m$ - tiimes differentiable function on $\mathbb{\mathbb { T }} k^{m}, t \in \mathbb{T}$.

Let $s \in \mathbb{T} k^{m-1}$, then 


$$
g(t)=\sum_{j j=0}^{m-1} h_{j j}(t, s) \Delta^{\Delta j}(s)+R_{m}(t)
$$

is called Taylors formula and the remainder term $R_{m}(t)$ is defined by

$$
R_{m}(t)=\int_{s}^{t} g^{\Delta^{m}}(r) h_{m-1}(t, \sigma(r)) \Delta r
$$

and it tends to zero as $m \rightarrow \infty$.

Proposition 2.1 [8] Let $g: \mathbb{T} \rightarrow \mathbb{R}$ is an analytic function at $s$ and at all $t \in(s-\varepsilon \varepsilon,+\infty) \cap \quad \mathbb{T}$ holds that $g(t)=\sum_{j j=0}^{\infty} a_{j j} h_{j j}(t, s)$. Then $g(t)$ is infinitely $j j$-times differentiable at $s$ and $g^{\Delta \Delta^{j j}}(s)=a_{i j}$

Theorem 2.2 [24] For any $t, s \in \mathbb{T T}$ with $\mu^{\Delta \Delta}=\zeta \zeta>0$ a constant. Then the product of monomials $h_{c}$ and $h_{\kappa \kappa}$ as follows

$$
\underset{c}{h}(t, s) \underset{\kappa \kappa}{h}(t, s)=\sum_{u=\kappa \kappa}^{c+\kappa \kappa} F(u, \kappa, r) \underset{c+\kappa \kappa-u}{\sigma \sigma \sigma^{\kappa \kappa}}(s, s) h_{u}(t, s)
$$

such that

$$
\begin{aligned}
& F(u, \kappa, r)=\begin{array}{lll}
\sum_{\omega=1}^{\kappa \kappa} & \sum_{l=1}^{u-\kappa \kappa}(-1)^{c-1} \varphi_{l}(u) u^{l(\omega+1)-} \frac{r(r+1)}{2}, & \text { ffor } u>r \\
1, & \text { ffor } u=\kappa
\end{array} \\
& \varphi_{l}(u, r)=\prod_{\substack{s \neq l \\
1 \leq s \leq r}} \frac{1}{\left(u^{-u}-u^{-s}\right)}, \varphi_{1}(u)=1 \text { and } u=1+\zeta \zeta
\end{aligned}
$$

Remark 2.1 $F(u, \kappa, r)$ in theorem(2.2) can be computed in another way according to the following formula [24]

$$
F(u, \kappa, r)=\sum_{j j_{1}=0}^{u-\kappa \kappa} \sum_{j j_{2}=j j_{1}}^{u-\kappa \kappa} \sum_{j j_{3}=j j_{2}}^{u-\kappa \kappa} \cdots \sum_{j j_{k \kappa-1}=j j_{k \kappa-2}}^{u-\kappa \kappa} \sum_{j j_{k \kappa}=j j_{k k-1}}^{u-\kappa \kappa} u^{\sum_{u=1}^{\kappa \kappa} j j_{u}}
$$

\section{The qq - differential transform method}

In 1986, Zhou proposed the DTM and applied it to analyze the electric circuit problems [43]. Inspired Zhou's idea and based on q-Taylor's formula, the q-DTM has been introduced [20]. In 2011, ElShahed has been extended the q-DTM to two dimensional for solving partial q-DEs [13]. In the same year, the damped q-DEs with strongly nonlinear has been used successfully by using the q-DTM [23]. This section devoted to derive some important formula related to DTM. Now, let $\psi \psi(t)$ be is $N$-tiimes q-differentiable on $\overline{q^{\mathbb{N}}}$, then by using theorem (2.1) with $t_{0}$ one can approximate the function $\psi \psi(t)$ as follows:

$$
\Psi(t)=\sum_{u=0}^{\infty} \Psi_{q}[u] h_{u}(t, 0)
$$

where

$$
\Psi_{q}[u]=\Psi^{\Delta^{u}}(0), \forall u=0,1,2, \ldots
$$

The Eq.((20)) is called the DTM, while Eq. ((19)) is called inverse of DTM.

Suppose that the functions $\Phi(t), \Psi(t)$, and $\Xi(t)$ are approximate as $\Phi(t)=\sum_{\kappa \kappa=0}^{\infty} \Phi_{q}[\kappa] h_{k \kappa}(t, 0)$, $\Psi(t)=\sum_{\kappa \kappa=0}^{\infty} \Psi_{q}[\kappa] h_{k \kappa}(t, 0)$, and $\Xi(t)=\sum_{\kappa \kappa=0}^{\infty} \Xi_{q}[\kappa] h_{k \kappa}(t, 0)$ respectively, then the essential mathematical operations achieved by DTM are presented in the next theorems. 
Theorem 3.1 For any real constants $a$, and $b$, if $=(t)=a \phi \phi(t) \mp b T(t)$, then $\vec{a}_{q}[\kappa]=a \phi_{q}[\kappa] \mp$ $b T_{q}[\kappa], \forall \kappa=0,1,2, \ldots$

Lemma 3.1 If $0 \in \mathbb{T T}$ and $\mu^{\Delta \Delta}=\zeta \zeta$ is nonzero constant, then multiplying any two monomials, $h_{c}(t, 0)$ and $h_{\kappa \kappa}(t, 0)$, is given as follows:

$$
h_{c}(t, 0) h_{\kappa \kappa}(t, 0)=F(r+\kappa, \kappa, r) h_{c+\kappa \kappa}(t, 0), \quad \kappa, r \neq 0, \quad \forall t \in \mathbb{T T}
$$

Proof. Since $\sigma^{m}(0)=0$ for all $m=0,1,2, \ldots$, we have

$$
\begin{array}{cc}
1, & r=0 \\
h_{c}{ }^{m}(0,0)=\emptyset, & \text { o.w. }, \quad \forall m=0,1,2, \ldots
\end{array}
$$

Using theorem(2.2), one can get

$$
\underset{c}{h}(t, 0) \underset{\kappa \kappa}{h}(t, 0)=\sum_{u=\kappa \kappa}^{c+m} F(u, \kappa, r) h_{c+\kappa \kappa-u}^{\sigma^{\kappa \kappa}}(0,0) h_{u}(t, 0),
$$

The result can be get it by substitute Eq.((22)) in Eq.((23)).

Theorem 3.2 If $T(t)==\Delta \Delta(t)$, then $T_{q}[\kappa]==\vec{q}[\kappa+1], \forall \kappa=0,1,2, \ldots$

Theorem 3.3 If $T(t)==(t) \phi \phi(t)$, then

$$
\begin{aligned}
& \Psi_{q}[0]=\Xi_{q}[0] \Phi_{q}[0] \\
& \Psi_{q}[1]=\Xi_{q}[1] \Phi_{q}[0]+\Xi_{q}[0] \Phi_{q}[1] \\
& \Psi_{q}[r]=\Xi_{q}[r] \Phi_{q}[0]+\Xi_{q}[0] \Phi_{q}[r]+\sum_{\kappa \kappa=1}^{c-1} \Xi_{q}[r-\kappa] \Phi_{q}[\kappa] F(r, r-\kappa, \kappa), r=2,3,4, \cdots
\end{aligned}
$$

Proof. Let $\Psi(t)=\Xi(t) \Phi(t)$ so one can have

$$
\begin{aligned}
& \sum_{\kappa \kappa=0}^{\infty} \Psi_{q}[\kappa] h_{k \kappa}(t, 0)=\sum_{\kappa \kappa=0}^{\infty} \Xi_{q}[\kappa] h_{k \kappa}(t, 0) \sum_{\kappa \kappa=0}^{\infty} \Phi_{q}[\kappa] h_{k \kappa}(t, 0) \\
& =\sum_{v=0}^{\infty} \sum_{\kappa \kappa=0}^{\infty} \Xi_{q}[w] \Phi_{q}[\kappa] h_{k \kappa}(t, 0) h_{v v}(t, 0)
\end{aligned}
$$

By using lemma(3.1), one can have

$$
\begin{aligned}
& \sum_{\kappa \kappa=0}^{\infty} \Psi_{q}[\kappa] h_{k \kappa}(t, 0)=\sum_{\kappa \kappa=0}^{\infty} \Xi_{q}[\kappa] \Phi_{q}[0] h_{\kappa k}(t, 0)+\sum_{\kappa \kappa=1}^{\infty} \Xi_{q}[0] \Phi_{q}[\kappa] h_{\kappa k}(t, 0) \\
& +\sum_{c=1}^{\infty} \sum_{\kappa \kappa=1}^{\infty} \Xi_{q}[r] \Phi_{q}[\kappa] F(\kappa+r, r, \kappa) h_{\kappa \kappa+c}(t, 0)
\end{aligned}
$$

Now, change the index in the third sum of Eq.((24)), we have

$$
\begin{aligned}
& \sum_{\kappa \kappa=0}^{\infty} \Psi_{q}[\kappa] h_{k \kappa}(t, 0)=\sum_{\kappa \kappa=0}^{\infty} \Xi_{q}[\kappa] \Phi_{q}[0] h_{k \kappa}(t, 0)+\sum_{\kappa \kappa=1}^{\infty} \Xi_{q}[0] \Phi_{q}[\kappa] h_{k \kappa}(t, 0) \\
& +\sum_{c=2}^{\infty} \sum_{\kappa \kappa=1}^{c-1} \Xi_{q}[r-\kappa] \Phi_{q}[\kappa] F(r, r-\kappa, \kappa) h_{c}(t, 0)
\end{aligned}
$$

Finally, the coefficients of $h_{c}(t, 0)$ are compared, and the result is obtained directly.

Theorem 3.4 If $f f(t)$ is analytic function on time scale $\mathbb{T} \mathbb{T}=\overline{q^{\mathbb{N}}}$ and $T(t)=f f(t) \phi \phi(t)$, then

$$
\Psi_{q}[0]=f f(0) \Phi_{q}[0]
$$




$$
\begin{aligned}
& \Psi_{q}[1]=f f \Delta(0) \Phi_{q}[0]+f f(0) \Phi_{q}[1] \\
& \Psi_{q}[r]=f f^{r}(0) \Phi_{q}[0]+f f(0) \Phi_{q}[r]+\sum_{\kappa \kappa=1}^{c-1} f f \Delta^{r-\kappa \kappa} \\
& (0) \Phi_{q}[\kappa] F(r, r-\kappa, \kappa), \quad r=2,3,4, \cdots
\end{aligned}
$$

Proof. Since $f f(t)$ is analytic function on time scale $\mathbb{T} T=\overline{q^{\mathbb{N}}}$, one can get $f f(t)=\sum_{\kappa \kappa=0}^{\infty} f f \Delta^{k \kappa}(0) h_{k k}(t, 0)$. Therefore, the result can be obtained directly using theorem(3.3).

Theorem 3.5 Ifff $(t)$ is analytic function on time scale $\mathbb{T} \mathbb{T}=\overline{q^{\mathbb{N}}}$ and $T(t)=f f(t) \phi \phi^{2}(t)$, then

$$
\begin{aligned}
& \Psi_{q}[0]=f f(0) \Phi_{q}^{2}[0] \\
& \Psi_{q}[1]=f f \Delta(0) \Phi_{q}^{2}[0]+2 f f(0) \Phi_{q}[1] \Phi_{q}[0] \\
& \Psi_{q}[r]=\Delta^{r}(0) \Phi_{q}^{2}[0]+f f(0) \Phi_{q}[r] \Phi_{q}[0]+f f(0) \Phi_{q}[0] \Phi_{q}[r]+f f(0){ }_{\kappa \kappa=1}^{c-1} \Phi_{q}[r- \\
& \kappa] \Phi_{q}[\kappa] F(r, r-\kappa, \kappa) \\
& f f
\end{aligned}
$$

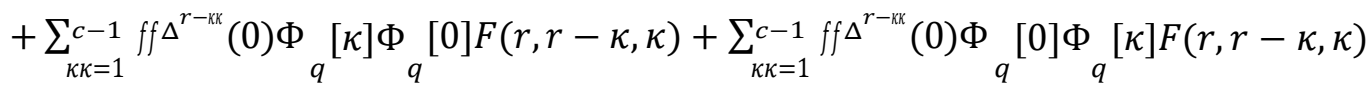

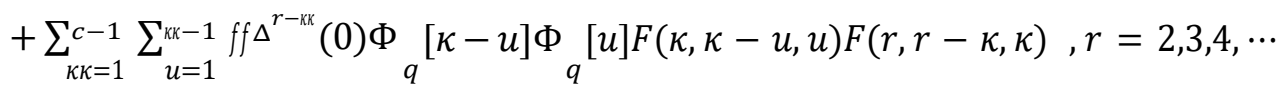

Proof. According to theorem (3.3), we find $\Phi^{2}(t)=\sum_{c=0}^{\infty} \Upsilon_{q}[r] h_{c}(t, 0)$

Where $\Upsilon_{q}[r]$ define as follows:

$$
\begin{aligned}
& \Upsilon_{q}[0]=\Phi_{q}^{2}[0] \\
& \Upsilon_{q}[1]=2 \Phi_{q}[1] \Phi_{q}[0] \\
& \Upsilon_{q}[r]=\Phi_{q}[r] \Phi_{q}[0]+\Phi_{q}[0] \Phi_{q}[r]+\sum_{\kappa \kappa=1}^{c-1} \Phi_{q}[r-\kappa] \Phi_{q}[\kappa] F(r, r-\kappa, \kappa), r=2,3,4, \cdots
\end{aligned}
$$

However, since $f f(t)$ is analytic function on time scale $\mathbb{T} \mathbb{T}=\overline{q^{\mathbb{N}}}$, we can get $f f(t)=\sum_{\kappa \kappa=0}^{\infty} f f \Delta^{k \kappa}(0) h_{k \kappa}(t, 0)$.

Using theorem(3.4), we have

$$
\begin{aligned}
& \Psi_{q}[0]=f f(0) \Upsilon_{q}[0] \\
& \Psi_{q}[1]=f f \Delta(0) \Upsilon_{q}[0]+f f(0) \Upsilon_{q}[1] \\
& \Psi_{q}[r]=f f^{\Delta^{r}}(0) \Upsilon_{q}[0]+f f(0) \Upsilon_{q}[r]+\sum_{\kappa \kappa=1}^{c-1} f f \Delta^{r-\kappa \kappa} \\
&
\end{aligned}
$$

Now, replace the values of $\Upsilon_{q}[r]$ in the above equations by its equivalent values in terms $\Phi_{q}[r]$, we get the result directly.

\section{Illustrated Examples}

Example 4.1 Consider the Riccati q-difference equation as follows:

$$
\begin{aligned}
& \Psi \Delta(t)=1-\Psi(t)^{2} \\
& \Psi(0)=0
\end{aligned}
$$

When $q$ tends to 1 , the solution exactly has the form 


$$
\Psi(t)=\tanh (t)
$$

Applying DTM to Eq.((26)) , we have

$2,3,4, \cdots$

$$
\begin{aligned}
& \Psi_{q}[1]+\Psi_{q}^{2}[0]-1=0 \\
& \Psi_{q}[2]+2 \Psi_{q}[1] \Psi_{q}[0]=0 \\
& \Psi_{q}[r+1]=-\Psi_{q}[r] \Psi_{q}[0]-\Psi_{q}[0] \Psi_{q}[r]-\sum_{\kappa \kappa=1}^{c-1} \Psi_{q}[r-\kappa] \Psi_{q}[\kappa] F(r, r-\kappa, \kappa), r=
\end{aligned}
$$
(29)

Again apply DTM to the initial conditions in Eq.((27)) , one can have

$$
\Psi_{q}[0]=0 .
$$

Using the Maple software, one can solve the recurrence relation in Eq.((29)) with Eq.((30)) to have the value of the unknown coefficients as follows:

$$
\begin{aligned}
& \Psi_{q}[1]=1 \\
& \Psi_{q}[2]=0 \\
& \Psi_{q}[3]=-3+q \\
& \Psi_{q}[4]=0 \\
& \Psi_{q}[5]=2\left(q^{2}-4 q+5\right)(-3+q)^{2} \\
& \Psi_{q}[6]=0 \\
& \Psi_{q}[7]=(-3+q)^{3}\left(q^{2}-3 q+3\right)\left(q^{4}-9 q^{3}+35 q^{2}-69 q+59\right)\left(q^{2}-4 q+5\right) \\
& \Psi_{q}[8]=0 \\
& \Psi_{q}[9]=2\left(q^{2}-4 q+5\right)^{2}\left(q^{2}-3 q+3\right)(-3+q)^{4}\left(2 q^{6}-26 q^{5}+143 q^{4}-427 q^{3}\right. \\
& \left.+737 q^{2}-711 q+313\right)\left(q^{4}-8 q^{3}+24 q^{2}-32 q+17\right)
\end{aligned}
$$

So, $\Psi(t) \simeq \sum_{c=0}^{9} \Psi_{q}[r] h_{c}(t, 0)$ is the first ten terms of the solution of this problem. Moreover, when $q \rightarrow 1$ this solution is given by

$$
\lim _{q \rightarrow 1} \Psi(t)=t-\frac{1}{3} t^{3}+\frac{2}{15} t^{5}-\frac{17}{315} t^{7}+\frac{62}{2835} t^{9}+\cdots
$$

When $q \rightarrow 1$, the solution in Eq.((31)) agrees exactly with the Taylor series of the given solution. Also, Eq.((31)) is agreement with the result in, Example(1) [37].

Example 4.2 Consider the Riccati q-difference equation as follows:

$$
\begin{aligned}
& \Psi \Delta(t)=1+2 \Psi(t)-\Psi^{2}(t) \\
& \Psi(0)=0
\end{aligned}
$$


When $q$ tends to 1 , the solution exactly has the form

$$
\Psi(t)=\sqrt{2} \tanh \left(\sqrt{2 t}+\frac{1}{2} \log \left(\frac{\sqrt{2}-1}{\sqrt{2}+1}\right)\right)+1
$$

Applying DTM to Eq.((32)) , we have

$$
\begin{aligned}
& \Psi_{q}[1]=2 \Psi_{q}[0]-\Psi_{q}^{2}[0]+1 \\
& \Psi_{q}[2]=2 \Psi_{q}[1]-2 \Psi_{q}[1] \Psi_{q}[0] \\
& \Psi_{q}[r+1]=2 \Psi_{q}[r]-2 \Psi_{q}[r] \Psi_{q}[0]-\sum_{\kappa k=1}^{c-1} \Psi_{q}[r-\kappa] \Psi_{q}[\kappa] F(r, r-\kappa, \kappa), \forall r=2,3,4, \cdots \\
& (35)
\end{aligned}
$$

Again apply DTM to the initial conditions in Eq.((33)) , one can have

$$
\Psi_{q}[0]=0 .
$$

Using the Maple software, one can solve the recurrence relation in Eq.((35)) with Eq.((36)) to have the value of the unknown coefficients as follows:

$$
\begin{aligned}
& \Psi_{q}[1]=1 \\
& \Psi_{q}[2]=2 \\
& \Psi_{q}[3]=1+q \\
& \Psi_{q}[4]=-4 q^{2}+22 q-26 \\
& \Psi_{q}[5]=-2(q-3)\left(q^{3}-9 q^{2}+31 q-37\right) \\
& \Psi_{q}[6]=-4 q^{7}+56 q^{6}-352 q^{5}+1276 q^{4}-2832 q^{3}+3732 q^{2}-2536 q+548 \\
& \Psi_{q}[7]=q^{11}-q^{10}-239 q^{9}+3230 q^{8}-21721 q^{7}+91686 q^{6}-262608 q^{5}+524197 q^{4} \\
& -725540 q^{3}+669551 q^{2}-373085 q+95377 \\
& \Psi_{q}[8]=8 q^{15}-228 q^{14}+2956 q^{13}-22836 q^{12}+114738 q^{11}-375506 q^{10}+685838 q^{9} \\
& +144320 q^{8}-5305474 q^{7}+18573384 q^{6}-38811708 q^{5}+55564898 q^{4}-55575960 q^{3} \\
& +37593290 q^{2}-15612662 q+3034030 \\
& \Psi_{q}[9]=-20 q^{20}+888 q^{19}-18662 q^{18}+247048 q^{17}-2312130 q^{16}+16272346 q^{15}- \\
& +16286776898 q^{3}-6068996878 q^{2}+1427426744 q-158832042 \\
& +392904980 q^{13}-1403278564 q^{12}+4115164306 q^{11}-9966897976 q^{10}+ \\
& q^{9}
\end{aligned}
$$

$89406344 q^{14}$ $19979418384 q^{9}$ $30993249576 q^{4}$ 
Therefore $\Psi(t) \simeq \sum_{c=0}^{9} \Psi_{q}[r] h_{c}(t, 0)$ is the first ten terms of the solution of the given problem. When $q \rightarrow 1$ this solution is given by

$$
\lim _{q \rightarrow 1} \Psi(t)=t+t^{2}+\frac{1}{3} t^{3}-\frac{1}{3} t^{4}-\frac{7}{15} t^{5}-\frac{7}{45} t^{6}+\frac{53}{315} t^{7}+\frac{71}{315} t^{8}+\frac{197}{2835} t^{9}+\cdots
$$

When $q \rightarrow 1$, the solution in Eq.((37)) agrees exactly with the Taylor series of the given solution.

Example 4.3 Consider the Riccati q-difference equation as follows:

$$
\begin{aligned}
& \Psi \Delta(t)=2 \Psi^{2}(t)-t \Psi(t)+1 \\
& \Psi(0)=0
\end{aligned}
$$

When $q$ tends to 1 , the solution exactly has the form

$$
\Psi(t)=\frac{t}{1-t^{2}}
$$

Applying DTM to Eq.((38)), we have

$$
\begin{aligned}
& \Psi_{q}[1]=2 \Psi_{q}^{2}[0]+1 \\
& \Psi_{q}[2]=4 \Psi_{q}[1] \Psi_{q}[0]+\Psi_{q}[0] \\
& \Psi_{q}[r+1]=4 \Psi_{q}[r] \Psi_{q}[0]+\Psi_{q}[r-1] F(r, 1, r-1) \\
& +2 \sum_{\kappa \kappa=1}^{c-1} \Psi_{q}[r-\kappa] \Psi_{q}[\kappa] F(r, r-\kappa, \kappa), \forall r=2,3,4, \cdots
\end{aligned}
$$

Again apply DTM to the initial conditions in Eq.((39)) , one can have

$$
\Psi_{q}[0]=0 .
$$

Using the Maple software, one can solve the recurrence relation in Eq.((41)) with Eq.((42)) to have the value of the unknown coefficients as follows:

$$
\begin{aligned}
& \Psi_{q}[1]=1 \\
& \Psi_{q}[2]=0 \\
& \Psi_{q}[3]=9-3 q \\
& \Psi_{q}[4]=0 \\
& \Psi_{q}[5]=15\left(q^{2}-4 q+5\right)(q-3)^{2} \\
& \Psi_{q}[6]=0 \\
& \Psi_{q}[7]=-3\left(q^{2}-3 q+3\right)\left(q^{2}-4 q+5\right)\left(6 q^{4}-54 q^{3}+211 q^{2}-419 q+361\right)(q-3)^{3} \\
& -725540 q^{3}+669551 q^{2}-373085 q+95377 \\
& \Psi_{q}[8]=0
\end{aligned}
$$




$$
\begin{aligned}
& \Psi_{q}[9]=15\left(12 q^{6}-156 q^{5}+858 q^{4}-2562 q^{3}+4423 q^{2}-4271 q+1885\right)\left(q^{2}-3 q+3\right) \\
& \times\left(q^{4}-8 q^{3}+24 q^{2}-32 q+17\right)\left(q^{2}-4 q+5\right)^{2}(q-3)^{4} \\
& \vdots
\end{aligned}
$$

Therefore $\Psi(t) \simeq \sum_{c=0}^{9} \Psi_{q}[r] h_{c}(t, 0)$ is the first ten terms of the solution of the given problem. When $q \rightarrow 1$ this solution is given by

$$
\lim _{q \rightarrow 1} \Psi(t)=t+t^{3}+t^{5}+t^{7}+t^{9}+\cdots
$$

When $q \rightarrow 1$, the solution in Eq.((43)) agrees exactly with the Taylor series of the given solution.

\section{Conclusions}

In this study, we introduce the difference transform method (DTM) based on Taylor formula for any time scale with its delta derivative of graininess function is a nonzero positive constant. Riccati type $q$-difference equations on quantum calculus have been successfully solved and the results coincide exactly with the Taylor series of the exact solution when $q$ - tends to 1 . In fact, this method is applicable to solving any nonlinear difference equations on any time scale with $\mu^{\Delta}>0$.

\section{References}

[1] S. Abbasbandy, Iterated he's homotopy perturbation method for quadratic riccati differential equation, Applied Mathematics and Computation, 175 (2006), pp. 581-589.

[2] D. D. Alessandro, Invariant manifolds and projective combinations of solutions of the riccati differential equation, Linear Algebra and its Applications, 279 (1998), pp. 181-193.

[3] S. Balaji, Legendre wavelet operational matrix method for solution of fractional order riccati differential equation, Journal of the Egyptian Mathematical Society, 23 (2015), pp. 263-270.

[4] K. Balla, Asymptotic behavior of certain riccati difference equations, Computers \& Mathematics with Applications, 36 (1998), pp. 243-250.

[5] A. Beghi and D. D’alessandro, Discrete-time optimal control with control dependent noise and generalized riccati difference equations, Automatica, 34 (1998), pp. 1031-1034.

[6] R. Bellman and R. Vasudevan, Dynamic programming and solution of wave equations, in Wave Propagation, Springer Netherlands, 1986, pp. 259-309.

[7] M. Bohner and A. Peterson, Dynamic equations on time scales: An introduction with applications, Springer Science \& Business Media, 2001.

[8] M. Bohner and A. C. Peterson, Advances in dynamic equations on time scales, Springer Science \& Business Media, 2002.

[9] C. Bota and B. Căruntu, Analytical approximate solutions for quadratic riccati differential equation of fractional order using the polynomial least squares method, Chaos, Solitons \& Fractals, 102 (2017), pp. 339-345.

[10] W. Y. Chen and H. L. Saad, On the gosper-petkovšek representation of rational functions, Journal of Symbolic Computation, 40 (2005), pp. 955-963. 
[11] W. Y. C. Chen, H. L. Saad, and L. H. Sun, An operator approach to the al-salam-carlitz polynomials, Journal of Mathematical Physics, 51 (2010), p. 043502.

[12] M. Dehghan and A. Taleei, A compact split-step finite difference method for solving the nonlinear schr" odinger equations with constant and variable coefficients, Computer Physics Communications, 181 (2010), pp. 43-51.

[13] M. El-Shahed and M. Gaber, Two-dimensional q-differential transformation and its application, Applied Mathematics and Computation, 217 (2011), pp. 9165-9172.

[14] M. El-Shahed, M. Gaber, and M. Al-Yami, The fractional q-differential transformation and its application, Communications in Nonlinear Science and Numerical Simulation, 18 (2013), pp. 42-55.

[15] M. A. El-Tawil, A. A. Bahnasawi, and A. Abdel-Naby, Solving riccati differential equation using adomian's decomposition method, Applied Mathematics and Computation, 157 (2004), pp. 503-514.

[16] T. Ernst, The history of q-calculus and a new method, 2000.

[17] F. Geng, A modified variational iteration method for solving riccati differential equations, Computers \& Mathematics with Applications, 60 (2010), pp. 1868-1872.

[18] S. Georgiev, Fractional dynamic calculus and fractional dynamic equations on time scales, Springer, Cham, Switzerland, 2018.

[19] M. Jambu, Quantum calculus an introduction, in New Trends in Algebras and

Combinatorics, WORLD SCIENTIFIC, feb 2020.

[20] S.-C. Jing and H.-Y. Fan, q -taylor's formula with its q -remainder, Communications in Theoretical Physics, 23 (1995), pp. 117-120.

[21] A. Lavagno, Basic-deformed quantum mechanics, Reports on Mathematical Physics, 64 (2009), pp. 7991.

[22] H.-K. Liu, Application of a differential transformation method to strongly nonlinear damped qdifference equations, Computers \& Mathematics with Applications, 61 (2011), pp. 2555-2561.

[23] H.-K. Liu, Application of a differential transformation method to strongly non-

linear damped q-difference equations, Computers \& Mathematics with Applications, 61 (2011), pp. 25552561.

[24] H.-K. Liu, The formula for the multiplicity of two generalized polynomials on time scales, Applied Mathematics Letters, 25 (2012), pp. 1420-1425.

[25] A. P. Magnus, Special nonuniform lattice (snul) orthogonal polynomials on discrete dense sets of points, Journal of Computational and Applied Mathematics, 65 (1995), pp. 253-265.

[26] A. Neirameh, Exact analytical solutions for 3d- gross-pitaevskii equation with periodic potential by using the kudryashov method, Journal of the Egyptian Mathematical Society, 24 (2016), pp. 49-53.

[27] G. D. Nicolao, On the time-varying riccati difference equation of optimal filtering, SIAM Journal on Control and Optimization, 30 (1992), pp. 1251-1269. 
[28] S. Nishioka, Differential transcendence of solutions of difference riccati equations and tietze's treatment, Journal of Algebra, 511 (2018), pp. 16-40.

[29] M. Nowakowski and H. C. Rosu, Newton's laws of motion in the form of a riccati equation, Physical Review E, 65 (2002).

[30] L. Ntogramatzidis and A. Ferrante, On the solution of the riccati differential equation arising from the LQ optimal control problem, Systems \& Control Letters, 59 (2010), pp. 114-121.

[31] K. Parand, S. A. Hossayni, and J. Rad, Operation matrix method based on bernstein polynomials for the riccati differential equation and volterra population model, Applied Mathematical Modelling, 40 (2016), pp. 993-1011.

[32] W. Reid, Riccati differential equations, Academic Press, New York, 1972.

[33] H. C. Rosu, S. C. Mancas, and P. Chen, Barotropic FRW cosmologies with chiellini damping, Physics Letters A, 379 (2015), pp. 882-887.

[34] H. L. Saad and M. A. Abdlhusein, New application of the cauchy operator on the homogeneous rogersszegÃú polynomials, The Ramanujan Journal, 56 (2021), pp. 347-367.

[35] H. L. Saad and F. A. Reshem, The operator g(a; b;dq) for the polynomials wn(x; y; a; b; q), Journal of Advances in Mathematics, 9 (2013), pp. 2888-2904.

[36] H. L. Saad and A. A. Sukhi, Another homogeneous q-difference operator, Applied Mathematics and Computation, 215 (2010), pp. 4332-4339.

[37] M. S. Semary and H. N. Hassan, The homotopy analysis method for q-difference equations, Ain Shams Engineering Journal, 9 (2018), pp. 415-421.

[38] J. Sugie, Nonoscillation theorems for second-order linear difference equations via the riccati-type transformation, II, Applied Mathematics and Computation, 304 (2017), pp. 142-152.

[39] H. Tietze,"Uber funktionalgleichungen, deren l" osungen keiner algebraischen differentialgleichung gen" ugen k" onnen, Monatsheftef" ur Mathematik und Physik, 16 (1905), pp. 329-364.

[40] G.-C. Wu, Variational iteration method for q-difference equations of second order, Journal of Applied Mathematics, 2012 (2012), pp. 1-5.

[41] M. I. Zelikin, Riccati equation in the classical calculus of variations, in Control Theory and Optimization I, Springer Berlin Heidelberg, 2000, pp. 60-79.

[42] H. Zhang and P. M. Dower, Max-plus fundamental solution semigroups for a class of difference riccati equations, Automatica, 52 (2015), pp. 103-110.

[43] J. Zhou, Differential Transformation and Its Applications for Electrical Circuits, Huazhong Univ. Press, Wuhan, China, 1986. 